\title{
Role of tumor suppressor molecules in genomic perturbations and damaged DNA repair involved in the pathogenesis of cancer and neurodegeneration (Review)
}

\author{
SATORU MATSUDA, MUTSUMI MURAKAMI, YUKA IKEDA, \\ YUKIE NAKAGAWA, AI TSUJI and YASUKO KITAGISHI \\ Department of Food Science and Nutrition, Nara Women's University, Nara 630-8506, Japan
}

Received December 14, 2018; Accepted May 11, 2020

DOI: $10.3892 /$ br.2020.1317

\begin{abstract}
Genomic perturbations due to inaccurate DNA replication, including inappropriate chromosomal segregation often underlie the development of cancer and neurodegenerative diseases. The incidence of these two diseases increases with age and exhibits an inverse association. Therefore, elderly subjects with cancer exhibit a reduced risk of a neurodegenerative disease, and vice versa. Both of these diseases are associated with aging and share several risk factors. Cells have multiple mechanisms to repair DNA damage and inaccurate replication. Previous studies have demonstrated that tumor suppressor proteins serve a critical role in the DNA damage response, which may result in genomic instability and thus induction of cellular apoptosis. Tumor suppressor genes, such as phosphatase and tensin homologue deleted on chromosome 10 (PTEN), breast cancer susceptibility gene 1 (BRCA1) and TP53 reduce genomic susceptibility to cancer by repairing the damaged DNA. In addition, these genes work cooperatively to ensure the inhibition of the development of several types of cancer. PTEN, BRCA1 and TP53 have been recognized as the most frequently deleted and/or mutated genes in various types of human cancer. Recently, tumor suppressor genes have also been shown to be involved in the development of neurodegenerative diseases. The present review summarizes the recent findings of the
\end{abstract}

Correspondence to: Professor Satoru Matsuda, Department of Food Science and Nutrition, Nara Women's University, Kita-Uoya Nishimachi, Nara 630-8506, Japan

E-mail:smatsuda@cc.nara-wu.ac.jp

Abbreviations: ALS, amyotrophic lateral sclerosis; ATM, ataxia telangiectasia mutated; BRCA1, breast cancer susceptibility gene 1; MDM2, murine double minute 2; PI3K, phosphoinositide-3 kinase; PTEN, phosphatase and tensin homologue deleted on chromosome 10; $\mathrm{Rb}$, retinoblastoma protein; ROS, reactive oxygen species; SODs, superoxide dismutases

Key words: BRCA1, PTEN, TP53, genomic stability, reactive oxygen species, DNA repair, cell signaling, carcinogenesis, neurodegeneration, aging functions of these tumor suppressors that are associated with genomic stability, and are involved in carcinogenic and neurodegenerative cell signaling. A summary is presented regarding the interactions of these tumor suppressors with their partners which results in transduction of downstream signals. The implications of these functions for cancer and neurodegenerative disease-associated biology are also highlighted.

\section{Contents}

1. Introduction

2. Reactive oxygen species (ROS), superoxide dismutase (SOD) and genomic instability in cancer and neurodegenerative diseases

3. Characteristics of tumor suppressor genes in cancer and neurodegenerative diseases

4. Functional interplay between tumor suppressor molecules in genomic-instability

5. Future perspectives

\section{Introduction}

Cells contain specific sensors to monitor DNA repair and induce apoptotic cell death when the DNA becomes damaged (1). Tumor suppressors serve critical roles in the regulation of the genomic integrity and the DNA repair pathways in various types of cells (2). Increased genomic instability can promote the development of cancer and neurodegenerative diseases, such as Alzheimer's disease (AD). The pathogenesis of these conditions is a multi-step process, accompanied by accumulation of genetic alterations in the somatic cells. In addition, aging contributes significantly to the impairment of physiological gene expression. AD and cancer are both prevalent in the elderly population (3). Although both are age-associated diseases, one is degenerative and the other is proliferative at the cellular level. Various factors that are upregulated to sustain cell growth in any type of cancer may be downregulated in AD-neurons contributing to neurodegeneration. Therefore, it is hypothesized that the inverse relationship between cancer and AD exhibits a reciprocal inverse association in several 
underlying aspects. Epidemiological data suggest that subjects who develop neurodegenerative diseases due to aging have a decreased risk of cancer (4). As neurodegeneration and carcinogenesis share a number of biological pathways, this inverse association is interesting to explore. The hypothesis proposes that neurodegeneration and carcinogenesis may manifest by several distinctive phenomena associated with senescence (5). The mechanism underlying intrinsic susceptibility of the cells towards either cell proliferative or cell apoptotic outcomes remain unknown (Fig. 1). A full understanding of these pathways may aid in the prevention and treatment of cancer or neurodegeneration (6). At present, effective treatment strategies for both diseases are lacking. The identification of the biological mechanisms responsible for the development of these diseases may provide novel targets for future therapeutic applications.

\section{Reactive oxygen species (ROS), superoxide dismutase (SOD) and genomic instability in cancer and neuro- degenerative diseases}

Cancer cells that originate from the primary site exhibit altered proliferative activity as a result of gene mutations responsible for controlling cellular proliferation. The mutations may result in the upregulation of proto-oncogenes and/or the downregulation of tumor suppressor genes $(7,8)$. It is commonly accepted that ROS are the primary trigger of these carcinogenic mutations (9). In addition, oxidative stress may contribute to cancer progression by affecting genomic instability (9). AD is one of the most notorious neurodegenerative diseases whose hallmarks include neuronal loss and/or dementia (10). The most significant DNA lesion affecting the progression of AD is likely caused by oxidative DNA damage (11). In general, increased levels of DNA damage and downregulation of cellular DNA repair capacity have been associated with age-associated diseases. Accumulation of genomic DNA damage can be caused by increased rates of oxidative-damage, which may exacerbate cancer and/or AD progression. Increased DNA oxidation has been reported in the post-mortem brain tissues of humans with AD (12). It has also been observed that DNA repair is dysregulated in AD (13). A stable increase in ROS levels can lead to potent induction of oxidative stress in cells, which causes genomic instability leading to the development of both diseases (Fig. 1).

The balance between ROS levels and the reducing equivalents in the cell determines the redox status and consequently, cell fate. For example, the therapeutic strategy in cancer should be based on treatments that increase ROS production and cause apoptotic cancer cell death (14). In contrast to this hypothesis, the strategy in treating neurodegenerative diseases should be based on protecting the activity of the neurons against the development of oxidative stress (15). Consequently, the redox status may have prognostic potential for the development of cancer and neurodegenerative diseases. The regulation of the redox status may affect the quality of life of the patients. The production of ROS is regulated by the action of chemical and enzymatic antioxidant systems, including ascorbate and the antioxidant enzymes, such as SOD. SOD enzymes possess a significant antioxidant role characterized by their ability to scavenge ROS (16). It has been demonstrated that
SOD defects are associated with the development of several types of disease $(17,18)$. Cytosolic SOD1 serves an important role in reducing the damage to the central nervous system (CNS) (19). Loss of SOD1 increases ROS levels, which triggers the induction of oxidative DNA damage to the cells. Mutations in the SODl gene are responsible for causing damage to the mitochondria and ultimately leading to the development of the progressive neurodegenerative diseases, such as familial amyotrophic lateral sclerosis (ALS) (20). It has also been revealed that SOD1-null animals develop specific age-related diseases, such as muscle atrophy (21). SOD2 has been identified by certain studies as a tumor suppressor since its expression is reduced in several types of cancer (22). In addition, the roles of SOD2 are involved in the development of neurodegenerative diseases (23). One of the most significant processes affected by SOD2 activity is the regulation of energy metabolism. Furthermore, SOD2 can protect mitochondrial DNA against oxidative damage. Mitochondrial adenosine triphosphate production activity has been reported to be impaired in AD (24). Mice containing one deleted copy of the SOD2 gene exhibit accelerated progression of AD development (25). SOD2 is localized within the mitochondrial matrix, which is the crucial site of free radical production from the electron transport chain (26). SOD3 exists as a secreted form in the extracellular matrix of several tissues. Downregulation of SOD3 has been shown to alter DNA copy number and/or to promote the hyper-methylation of the promoter DNA region (27). SOD3 is secreted to the extracellular matrix in the CNS tissues. Previous studies have shown that inhibition of ROS production by SOD activation may reduce neuronal cell death and glial cell activation, which may have an unusual effective therapeutic potential compared with conventional treatments $(28,29)$.

\section{Characteristics of tumor suppressor genes in cancer and neurodegenerative diseases}

Tumor suppressor gene products are molecules which may protect a cell from carcinogenesis (30-36). Accordingly, loss of function in these molecules may be important in the formation of several types of cancer $(30,34)$. Well-studied tumor suppressor molecules include TP53, breast cancer susceptibility gene 1 (BRCA1), phosphatase and tensin homologue deleted on chromosome 10 (PTEN), APC and the retinoblastoma protein ( $\mathrm{Rb})$, amongst others (31-36). Mutations in BRCA1 may increase the risk of the development of breast and ovarian cancer (30). Furthermore, it has been shown that the genetic variation in the BRCAl gene is associated with prostate cancer development (31). Activation of the phosphoinositide 3-kinase (PI3K) enzyme is often associated with the development of BRCA1-associated breast cancer (32). The functional insufficiency of BRCA1 activates the PI3K/AKT oncogenic pathway, which may also be associated with the development of neurodegenerative diseases (33). Reduced levels of BRCA1 have been found in the brains of patients with AD. In addition, knockdown of neuronal BRCA1 has been shown to affect synaptic impairment and memory deficits (34). Accordingly, BRCA1 may support neuronal integrity and cognitive functions, whereas the reduced function of the neuronal BRCA1 contributes to cognitive deficits in AD (35). 


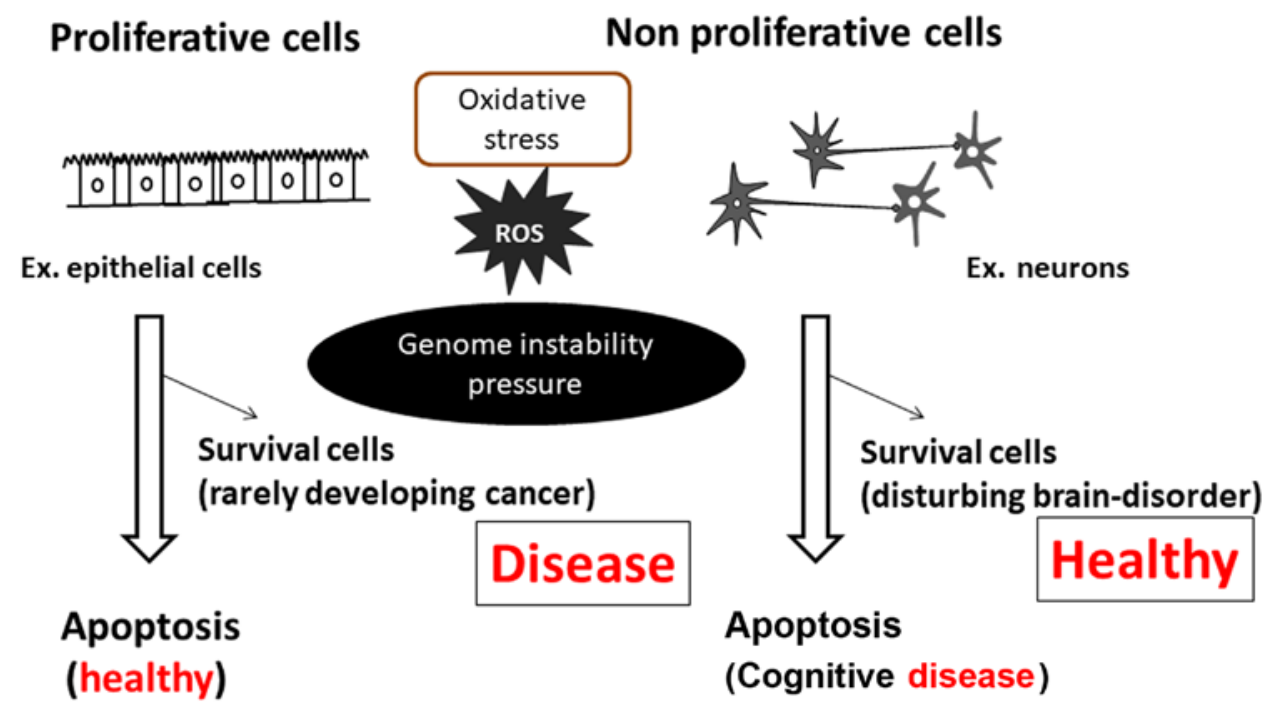

Because of regeneration by proliferation

Figure 1. Genomic instability-dependent cellular fates of proliferative and non-proliferative cells. Schematic illustration of the potential mechanisms underlying the involvement of oxidative stress in the genomic instability in proliferative and non-proliferative cells. In response to the genotoxic oxidative damage signals caused by ROS, the cellular fates of proliferative and non-proliferative cells may differ. Subsequently, this may result in activation of different molecular events leading to healthy or diseased states. ROS, reactive oxygen species; Ex. superoxide, hydroxyl radical, peroxides or singlet oxygen.

The changes to DNA repair as a result of BRCA1 mutations may occur early in the progression of neurodegeneration. BRCA1 can bind to BRCA2, Rad50, Rad51 and Rb in order to activate the cell cycle checkpoints (36). Although these protein complexes are present in neurons of the adult cortex and cerebellum, their expression is considerably diminished in the neurons of the brain tissues from AD subjects (37). These complexes may be involved in DNA repair and the regulation of the cell cycle checkpoints (38). The Rb protein is another tumor suppressor involved in cell-cycle regulation and neural cell apoptosis $(39,40) . \mathrm{Rb}$ is used as a potential diagnostic marker for $\mathrm{AD}(39,40)$. The $\mathrm{p} 27^{\mathrm{Kip} 1}$ and $\mathrm{p} 21^{\text {Waf1 }}$ proteins are also activated by BRCA1 in AD (41). In addition, mutations of the pathogenic protein presenilin in AD result in a specific increase in the expression levels of $\mathrm{p} 21^{\mathrm{Waf} 1}$ and in the expression levels of the proteins involved in TP53 signaling (42). The levels of $\mathrm{p} 21^{\text {Waf } 1}$ are increased in AD. The induction of cell death or cell survival is mediated by the coordinated action of the BRCA1, p21 ${ }^{\text {Waf1 }}$ and TP53 proteins dependent on the type of oxidative damage caused by the cells.

The PTEN gene is ubiquitously expressed during embryogenesis in mammals (43). PTEN is a tumor suppressor and its absence may exacerbate prognosis during the early stages of cancer (44). Furthermore, mutations of PTEN underlie certain types of hamartoma tumor syndromes (Cowden syndrome, PTEN-related Proteus syndrome, Proteus-like syndrome, Bannayan-Riley-Ruvalcaba syndrome). These mutations further increase the risk for the development of various types of cancer (45). PTEN deficiency induces a stress on DNA replication, which disturbs mitotic spindle formation leading to an increase in chromosomal instability (46). In addition, PTEN may protect genomic integrity by controlling multiple processes involved in chromosome inheritance-formation (46). Various somatic PTEN mutations in cancer have suggested an involvement of PTEN in the carcinogenesis process. Inactivation of PTEN affects the activation of PI3K/AKT signaling, which induces increased expression of several genes required for cell survival, growth and migration. These genes are all indispensable in cancer development. In contrast to these observations, the induction of PTEN may be associated with the activation of a pathway required for cell apoptosis $(47,48)$. Therefore, neuronal cell death has been attributed, at least in part, to the alterations in the function of PTEN, whereas the inhibition of PTEN may retain synaptic function and improve cognition in AD, as demonstrated in animal models $(49,50)$. Furthermore, overexpression of PTEN results in synaptic depression which mimics the symptoms of $A \beta$-induced $A D(51)$. It is interesting to note that $A \beta$ recruits PTEN and results in the accumulation of its levels in the postsynaptic compartment (52). The repression of PTEN and/or the stimulation of AKT are important in the protection of the neuron. Furthermore, decreased expression of PTEN may result in an increase in mitochondrial activity associated with high levels of ROS production (53). Extensive induction of oxidative stress caused by environmental factors can result in neurological diseases, including ALS (54). PTEN and PTEN-induced putative kinase 1 may be involved in the pathways of the regulation of cellular oxidative stress. The maintenance of the balance between pro-oxidants and anti-oxidants is required for healthy neurons.

TP53 is a transcription factor ubiquitously expressed in all cell types, which regulates the cell cycle checkpoints and the induction of apoptosis following DNA damage (55). In response to various cellular stresses, activated TP53 may induce cell cycle arrest (56). Failure of the DNA repair machinery to correct extensive DNA damage activates the induction of apoptotic cell death mediated by the TP53 protein (56). The genomic integrity is maintained by the cells decision to induce DNA repair or to activate the apoptotic cascade (57). The TP53 gene is frequently mutated in numerous types of cancer cells, suggesting that it serves a critical role in preventing normal cells from malignant transformation (58). The importance of TP53 as an inherited cancer susceptibility gene product has 


\section{A}

In the young

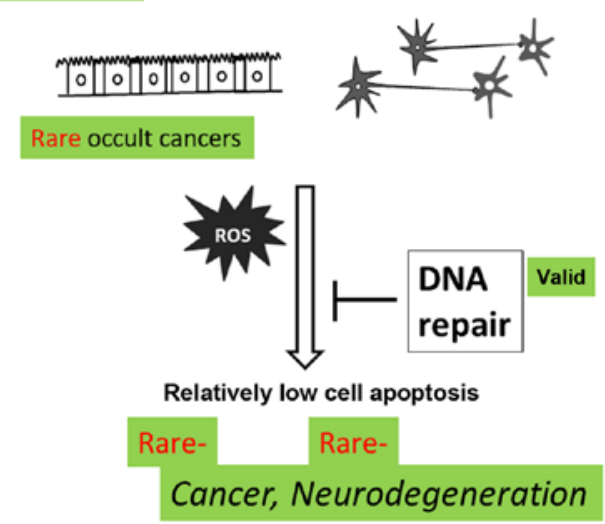

\section{B In the elderly}

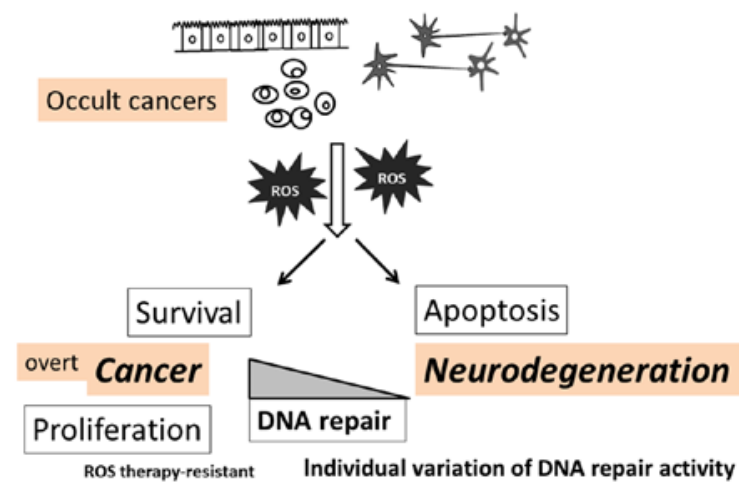

Figure 2. Induction of survival or apoptosis in age-related diseases Determination of either survival or apoptosis may be dependent on the balance between the DNA damage and DNA repair processes. Individua variations in the DNA repair activity in combination with the levels of DNA damage may determine disease status in the elderly; specifically cancer or neurodegenerative disease. In younger individuals, the absence of cancer may be associated with effective DNA repair activity in proliferative and non-proliferative neurons. (A) In contrast to younger individuals, excess amounts of DNA damage may result in the development of cancer in the elderly. High levels of DNA repair activity in cells results in cancer survival, reduced neurodegeneration and induction of apoptosis; (B) whereas the opposite effect is noted in the case of low levels of DNA repair activity in neurons.

been demonstrated in Li-Fraumeni syndrome, and is associated with a high risk of developing cancer in multiple types of malignancies (59). The functions of TP53 are supported by different downstream targets and several effectors. Among these, cyclin-dependent kinase (CDK) inhibitors such as p2 $1^{\text {Waf1 }}$ are important mediators of TP53 (60). As mentioned earlier, $\mathrm{p} 21^{\text {Waf1 }}$ is activated by BRCA1 in AD. Furthermore, the specific severity of the clinical progression of AD is associated with mutation of the presenilin protein into a pathogenic isoform that is frequently observed in patients with $\mathrm{AD}(42)$. This mutation may be mediated by $\mathrm{p} 21^{\text {Wafl }}$ and TP53 signaling proteins (42). Accumulation of DNA damage irrespective of the TP53 function may also lead to neuronal cell death following AD.

The comprehensive roles of these tumor suppressor signaling molecules can be used to explore the potential inverse associations between AD and cancer. Cell survival or apoptosis may be governed by the balance between DNA

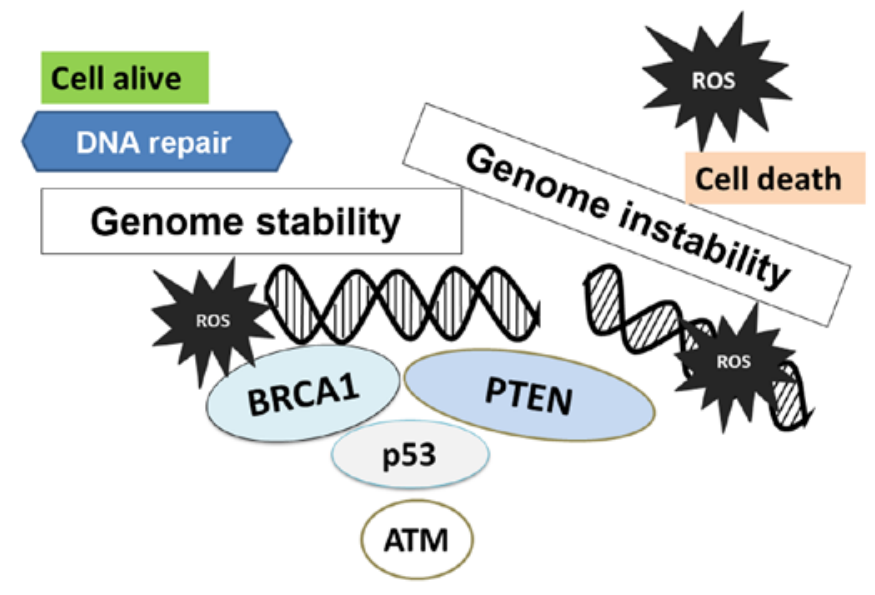

Figure 3. Schematic illustration depicting the equilibrium between genomic stability and instability. This balance can be sustained by the functions of several tumor suppressors. To maintain genomic stability, the cell machinery makes a decision of whether to induce DNA repair or apoptosis in damaged cells, which would result in cell survival or cell death. Specific critical functions have been omitted for clarity. ROS, reactive oxygen species.

damage and repair, which has received increasing attention as a major pathway used in the treatment of cancer and neurodegenerative diseases $(61,62)$.

\section{Functional interplay between tumor suppressor molecules in genomic-instability}

DNA repair is a multi-step process by which a cell corrects DNA damage (63-66). The DNA repair system firmly sustains genomic fidelity through the recognition and repair of the damaged nucleotides. Therefore, it is likely that carcinogenesis is principally caused by increased DNA damage, and that the dysregulated function of the DNA repair machinery can lead to decreased genomic stability. These phenomena may also be associated with neurodegenerative disorders including AD. As several tumor suppressors serve a crucial role in maintaining genomic stability, the dysregulated or reduced function of the tumor suppressors is associated with increased genomic instability in cells, which consequently accelerates the mutations of other critical genes (63). Deficiency in DNA damage responses and/or downregulation of the DNA repair mechanism results in increased genomic instability. The recognition of DNA damage may rely on the function of ataxia telangiectasia mutated (ATM), which is a cell-cycle checkpoint kinase that phosphorylates various proteins including BRCA1 and TP53 in response to DNA damage (64). BRCA1 associates with $\operatorname{Rad} 50$ and Rad51, two types of DNA recombinase enzymes required for genomic stability (65). BRCA1 activates CDK inhibitor p21 waf1 and TP53, which subsequently adjusts the expression levels of several proteins that control cell-cycle checkpoints, collectively decreasing cell-cycle progression (65). In addition, upregulation of PTEN represses the expression levels of AKT kinase and the activity of murine double minute 2 (MDM2), which enhances the levels of TP53, leading to the induction of cell-cycle arrest or apoptosis (66). MDM2 is an oncoprotein that regulates several tumorigenic proteins, whose mRNA levels are regulated by TP53 in response to DNA damage (66). 
MDM2 is also involved in the development of neurodegenerative diseases (66). One of the mechanisms by which PTEN induces cell-cycle arrest is by regulating AKT such that the levels of $\mathrm{p} 27^{\mathrm{Kip} 1}$ are increased (66). BRCA1 is associated with chromosomal stability and spindle formation (67). Previous studies have suggested that the proteins BRCA1 and TP53, and the PTEN and AKT signaling pathways are involved in modifying genomic stability and/or response to DNA damage $(66,67)$. These processes involve additional proteins that interact with each other in a complex network $(66,67)$. Nuclear localization of tumor suppressors mediates several activities of tumor suppression required for genomic stability, which may contain a range of functions including DNA repair and cell cycle arrest. For example, PTEN interacts with histone proteins and controls chromatin function (68). In addition, PTEN regulates the expression of Rad51, which reduces DNA damage (68). It has been suggested that PTEN serves a role in the protection of the cells following induction of oxidative damage (33). The activity of PTEN can be repressed by ROS and the loss of its activity is associated with several types of cancer, which could result from genomic instability $(69,70)$. Furthermore, decreased levels of PTEN are associated with reduced irradiation-resistance, which can be suppressed by ectopic PTEN expression (71). PTEN serves a role in the induction of cell-cycle arrest through activation of ATM signaling. Therefore, knockdown of PTEN increases ATM activation, decreasing the phosphorylation levels of ATM substrates, such as TP53 and BRCA1, which is considered a significant part of the DNA repair signaling cascade (72).

\section{Future perspectives}

Genomic integrity may be sustained through the function of several tumor suppressor genes, which reduces pathologic alterations, such as the development of carcinogenesis and neurodegeneration. Loss of the function of the tumor suppressor genes may reduce DNA repair and induce genomic instability, which can lead to cell apoptosis. Furthermore, it may enhance the sensitivity of cancer cells to irradiation. With regards to therapeutic applications, this treatment may be beneficial for patients with cancer. Neurodegenerative diseases can be caused by neuronal cell apoptosis of non-proliferative neuronal cells (Fig. 1). The use of alkylating agents and/or irradiation for cancer therapy may promote neurodegeneration due to the genomic instability caused from excess production of ROS (73). In conclusion, the present review reports on the increased number of age-related occult cancer and the reduced potential of anti-oxidative defense mechanisms that would determine the bipolar fates of patients with regard to the development of cancer or of neurodegenerative diseases in elderly individuals (Fig. 2). This may explain why the inverse association occurs in the elderly in contrast to younger patients (Fig. 2). In the absence of the excess ROS production, bacterial inflammation and/or life-style-associated fatty diseases may increase ROS levels in the elderly, which may in turn increase cancer incidence and the induction of neuronal apoptosis. Consequently, the extent of DNA repair capacity would determine the tendency to develop either cancer or neurodegenerative diseases (Fig. 2).
Tumor suppressor genes can act in a cooperative manner to maintain genomic integrity (Fig. 3). The understanding of the complete assembly of the tumor suppressor proteins can aid in the development of effective treatment approaches for cancer and neurodegenerative diseases. Further mechanistic studies are required in order to understand the precise assembly among tumor suppressor proteins and the molecular signaling mechanisms responsible for facilitating the development of effective treatments, which can regulate genomic stability and improve therapeutic efficacy. These factors may influence the development of neurodegeneration and/or regulate cognitive dysfunction in the elderly. Further studies are required to elucidate the biological mechanisms underlying the inverse association between cancer and AD with the goal of identifying preventative molecular targets.

\section{Acknowledgements}

Not applicable.

Funding

The present work was supported in part by JSPS KAKENHI (grant no. JP18K17964). In addition, funding was received in part by a grant from the Nara Women's University in Japan.

\section{Availability of data and materials}

Not applicable.

\section{Authors' contributions}

SM has contributed to the conception and design of the study. MM, YI, YN, AT, YK and SM participated in drafting and revising the article. All authors have read and approved the final manuscript.

\section{Ethics approval and consent to participate}

Not applicable.

\section{Patient consent for publication}

Not applicable.

\section{Competing interests}

The authors declare that they have no competing interests.

\section{References}

1. Awasthi P, Foiani M and Kumar A: ATM and ATR signaling at a glance. J Cell Sci 128: 4255-4262, 2015.

2. Van Haaften G, Plasterk RH and Tijsterman M: Genomic instability and cancer: Scanning the Caenorhabditis elegans genome for tumor suppressors. Oncogene 23: 8366-8375, 2004.

3. Ghezzi EM and Ship JA: Systemic diseases and their treatments in the elderly: Impact on oral health. J Public Health Dent 60: 289-296, 2000.

4. Zhang Q, Guo S, Zhang X, Tang S, Shao W, Han X, Wang L and Du Y: Inverse relationship between cancer and Alzheimer's disease: A systemic review meta-analysis. Neurol Sci 36: 1987-1994, 2015. 
5. Musicco M, Adorni F, Di Santo S, Prinelli F, Pettenati C, Caltagirone C, Palmer K and Russo A: Inverse occurrence of cancer and Alzheimer disease: A population-based incidence study. Neurology 81: 322-328, 2013,

6. Nudelman KN, Risacher SL, West JD, McDonald BC, Gao S and Saykin AJ: Alzheimer's disease neuroimaging initiative. Association of cancer history with Alzheimer's disease onset and structural brain changes. Front Physiol 5: 423, 2014.

7. Liu Y, Chen Q and Zhang JT: Tumor suppressor gene 14-3-3sigma is down-regulated whereas the proto-oncogene translation elongation factor 1delta is up-regulated in non-small cell lung cancers as identified by proteomic profiling. J Proteome Res 3: 728-735, 2004.

8. Boiko AD, Porteous S, Razorenova OV, Krivokrysenko VI, Williams BR and Gudkov AV: A systematic search for downstream mediators of tumor suppressor function of p53 reveals a major role of BTG2 in suppression of Ras-induced transformation. Genes Dev 20: 236-252, 2006.

9. Payne CM, Bernstein C, Dvorak K and Bernstein H: Hydrophobic bile acids, genomic instability, Darwinian selection, and colon carcinogenesis. Clin Exp Gastroenterol 1: 19-47, 2008.

10. Fujikake N, Shin M and Shimizu S: Association between autophagy and neurodegenerative diseases. Front Neurosci 12: $255,2018$.

11. Madabhushi R, Pan L and Tsai LH: DNA damage and its links to neurodegeneration. Neuron 83: 266-282, 2014

12. Bradley-Whitman MA, Timmons MD, Beckett TL, Murphy MP Lynn BC and Lovell MA: Nucleic acid oxidation: An early feature of Alzheimer's disease. J Neurochem 128: 294-304, 2014

13. Canugovi C, Shamanna RA, Croteau DL and Bohr VA: Base excision DNA repair levels in mitochondrial lysates of Alzheimer's disease. Neurobiol Aging 35: 1293-1300, 2014

14. Chen W, Zou P, Zhao Z, Chen X, Fan X, Vinothkumar R, Cui R, Wu F, Zhang Q, Liang G and Ji J: Synergistic antitumor activity of rapamycin and EF24 via increasing ROS for the treatment of gastric cancer. Redox Biol 10: 78-89, 2016

15. Zhao L, Wang JL, Wang YR and Fa XZ: Apigenin attenuates copper-mediated $\beta$-amyloid neurotoxicity through antioxidation, mitochondrion protection and MAPK signal inactivation in an AD cell model. Brain Res 1492: 33-45, 2013

16. Wang Y, Branicky R, Noë A and Hekimi S: Superoxide dismutases: Dual roles in controlling ROS damage and regulating ROS signaling. J Cell Biol 217: 1915-1928, 2018.

17. Pong K: Oxidative stress in neurodegenerative diseases: Therapeutic implications for superoxide dismutase mimetics. Expert Opin Biol Ther 3: 127-139, 2003

18. Nazıroğlu M, Muhamad S and Pecze L: Nanoparticles as potential clinical therapeutic agents in Alzheimer's disease: Focus on selenium nanoparticles. Expert Rev Clin Pharmacol 10: 773-782, 2017.

19. Cudkowicz ME, Pastusza KA, Sapp PC, Mathews RK, Leahy J, Pasinelli P, Francis JW, Jiang D, Andersen JK and Brown RH Jr: Survival in transgenic ALS mice does not vary with CNS glutathione peroxidase activity. Neurology 59: 729-734, 2002.

20. Sangwan S and Eisenberg DS: Perspective on SOD1 mediated toxicity in Amyotrophic Lateral Sclerosis. Postepy Biochem 62: 362-369, 2016.

21. Kostrominova TY: Advanced age-related denervation and fiber-type grouping in skeletal muscle of SOD1 knockout mice. Free Radic Biol Med 49: 1582-1593, 2010.

22. Kang SW: Superoxide dismutase 2 gene and cancer risk: Evidence from an updated meta-analysis. Int J Clin Exp Med 8 $14647-14655,2015$.

23. Flynn JM and Melov S: SOD2 in mitochondrial dysfunction and neurodegeneration. Free Radic Biol Med 62: 4-12, 2013

24. Hroudová J, Singh N and Fišar Z: Mitochondrial dysfunctions in neurodegenerative diseases: Relevance to Alzheimer's disease. Biomed Res Int 2014: 175062, 2014

25. Hinerfeld D, Traini MD, Weinberger RP, Cochran B, Doctrow SR, Harry J and Melov S: Endogenous mitochondrial oxidative stress: Neurodegeneration, proteomic analysis, specific respiratory chain defects, and efficacious antioxidant therapy in superoxide dismutase 2 null mice. J Neurochem 88: 657-667, 2004.

26. Papa L, Hahn M, Marsh EL, Evans BS and Germain D: SOD2 to SOD1 switch in breast cancer. Biol Chem 289: 5412-5416, 2014.

27. Teoh-Fitzgerald ML, Fitzgerald MP, Jensen TJ, Futscher BW and Domann FE: Genetic and epigenetic inactivation of extracellular superoxide dismutase promotes an invasive phenotype in human lung cancer by disrupting ECM homeostasis. Mol Cancer Res 10: 40-51, 2012.
28. Baluchnejadmojarad T, Mansouri M, Ghalami J, Mokhtari Z and Roghani M: Sesamin imparts neuroprotection against intrastriatal 6-hydroxydopamine toxicity by inhibition of astroglial activation, apoptosis, and oxidative stress. Biomed Pharmacother 88: 754-761, 2017.

29. Petro M, Jaffer H, Yang J, Kabu S, Morris VB and Labhasetwar V: Tissue plasminogen activator followed by antioxidant-loaded nanoparticle delivery promotes activation/mobilization of progenitor cells in infarcted rat brain. Biomaterials 81: 169-180, 2016.

30. Dilawari A, Cangiarella J, Smith J, Huang A, Downey A and Muggia F: Co-existence of breast and ovarian cancers in BRCA germ-line mutation carriers. Ecancermedicalscience 2: 109, 2008.

31. Modena A, Iacovelli R, Scarpa A, Brunelli M, Ciccarese C, Fantinel E, Bimbatti D, Massari F, Martignoni G and Tortora G Investigating BRCA mutations: A breakthrough in precision medicine of castration-resistant prostate cancer. Target Oncol 11: 569-577, 2016.

32. Guirouilh-Barbat JK, Wilhelm T and Lopez BS: AKT1/BRCA1 in the control of homologous recombination and genetic stability: The missing link between hereditary and sporadic breast cancers. Oncotarget 1: 691-699, 2010.

33. Ogino M, Ichimura M, Nakano N, Minami A, Kitagishi Y and Matsuda S: Roles of PTEN with DNA repair in Parkinson's disease. Int J Mol Sci 17: 954, 2016.

34. Gonzalez ME, Li X, Toy K, DuPrie M, Ventura AC, Banerjee M, Ljungman M, Merajver SD and Kleer CG: Downregulation of EZH2 decreases growth of estrogen receptor-negative invasive breast carcinoma and requires BRCA1. Oncogene 28: 843-853, 2009.

35. Suberbielle E, Djukic B, Evans M, Kim DH, Taneja P, Wang X, Finucane M, Knox J, Ho K, Devidze N, et al: DNA repair factor BRCA1 depletion occurs in Alzheimer brains and impairs cognitive function in mice. Nat Commun 6: 8897, 2015.

36. Brown EJ: Analysis of cell cycle progression and genomic integrity in early lethal knockouts. Methods Mol Biol 280: 201-212, 2004.

37. Jacobsen E, Beach T, Shen Y, Li R and Chang Y: Deficiency of the Mre11 DNA repair complex in Alzheimer's disease brains. Brain Res Mol Brain Res 128: 1-7, 2004.

38. Jhanwar-Uniyal M: BRCA1 in cancer, cell cycle and genomic stability. Front Biosci 8 (Suppl): S1107-S1117, 2003.

39. Hradek AC, Lee HP, Siedlak SL, Torres SL, Jung W, Han AH and Lee HG: Distinct chronology of neuronal cell cycle re-entry and tau pathology in the 3xTg-AD mouse model and Alzheimer's disease patients. J Alzheimers Dis 4: 57-65, 2015.

40. Padmanabhan J, Brown K and Shelanski ML: Cell cycle inhibition and retinoblastoma protein overexpression prevent Purkinje cell death in organotypic slice cultures. Dev Neurobiol 67: 818-826, 2007.

41. Delobel P, Lavenir I, Ghetti B, Holzer M and Goedert M: Cell-cycle markers in a transgenic mouse model of human tauopathy: Increased levels of cyclin-dependent kinase inhibitors p21Cip1 and p27Kip1. Am J Pathol 168: 878-887, 2006.

42. Bialopiotrowicz E, Szybinska A, Kuzniewska B, Buizza L, Uberti D, Kuznicki J and Wojda U: Highly pathogenic Alzheimer's disease presenilin 1 P117R mutation causes a specific increase in p53 and p21 protein levels and cell cycle dysregulation in human lymphocytes. J Alzheimers Dis 32: 397-415, 2012.

43. Yin Y and Shen WH: PTEN: A new guardian of the genome. Oncogene 27: 5443-5453, 2008.

44. Wise HM, Hermida MA and Leslie NR: Prostate cancer, PI3K, PTEN and prognosis. Clin Sci (Lond) 131: 197-210, 2017.

45. Yakubov E, Ghoochani A, Buslei R, Buchfelder M, Eyüpoglu IY and Savaskan N: Hidden association of Cowden syndrome, PTEN mutation and meningioma frequency. Oncoscience 3: 149-155, 2016.

46. Hou SQ, Ouyang M, Brandmaier A, Hao H and Shen WH: PTEN in the maintenance of genome integrity: From DNA replication to chromosome segregation. Bioessays: 39: 1700082, 2017.

47. Ming $M$ and He YY: PTEN in DNA damage repair. Cancer Lett 319: 125-129, 2012.

48. Fiandalo MV and Kyprianou N: Caspase control: Protagonists of cancer cell apoptosis. Exp Oncol 34: 165-175, 2012.

49. Krishnan A and Zochodne DW: Is cytoplasmic PTEN a specific target for neuronal survival? Mol Neurobiol 52: 1758-1764, 2015

50. Asua D, Bougamra G, Calleja-Felipe M, Morales M and Knafo S: Peptides acting as cognitive enhancers. Neuroscience 370: 81-87, 2018.

51. Frere S and Slutsky I: Targeting PTEN interactions for Alzheimer's disease. Nat Neurosci 19: 416-418, 2016. 
52. Knafo S, Sánchez-Puelles C, Palomer E, Delgado I, Draffin JE, Mingo J, Wahle T, Kaleka K, Mou L, Pereda-Perez I, et al: PTEN recruitment controls synaptic and cognitive function in Alzheimer's models. Nat Neurosci 19: 443-453, 2016.

53. Zhang YY, Huang J, Yang M, Gu LJ, Ji JY, Wang LJ and Yuan WJ: Effect of a low-protein diet supplemented with Keto-acids on autophagy and inflammation in 5/6 nephrectomized rats. Biosci Rep 35: e00263, 2015.

54. Estevez AO, Morgan KL, Szewczyk NJ, Gems D and Estevez M: The neurodegenerative effects of selenium are inhibited by FOXO and PINK1/PTEN regulation of insulin/insulin-like growth factor signaling in Caenorhabditis elegans. Neurotoxicology 41 : 28-43, 2014.

55. Machado-Silva A, Perrier S and Bourdon JC: p53 family members in cancer diagnosis and treatment. Semin Cancer Biol 20: 57-62, 2010.

56. Stewart-Ornstein J and Lahav G: p53 dynamics in response to DNA damage vary across cell lines and are shaped by efficiency of DNA repair and activity of the kinase ATM. Sci Signal 10 eaah6671, 2017.

57. Zhang XP, Liu F, Cheng Z and Wang W: Cell fate decision mediated by p53 pulses. Version 2. Proc Natl Acad Sci USA 106: 12245-50, 2009.

58. Bénard J, Douc-Rasy S and Ahomadegbe JC: TP53 family members and human cancers. Hum Mutat 21: 182-191, 2003.

59. Varley JM: Germline TP53 mutations and Li-Fraumeni syndrome. Hum Mutat 21: 313-320, 2003.

60. Zhao J,Lammers P, Torrance CJ and Bader AG:TP53-independent function of miR-34a via HDAC1 and p21(CIP1/WAF1.). Mol Ther 21: 1678-1686, 2013.

61. O'Neil N and Rose A: DNA repair. WormBook. Jan, 13, 1-12, 2006.

62. Golubnitschaja O: Cell cycle checkpoints: The role and evaluation for early diagnosis of senescence, cardiovascular, cancer and neurodegenerative diseases. Amino Acids 32: 359-371, 2007

63. Hazan I, Hofmann TG and Aqeilan RI: Tumor suppressor genes within common fragile sites are active players in the DNA damage response. PLoS Genet 12: e1006436, 2016.

64. Thurn KT, Thomas S, Raha P, Qureshi I and Munster PN Histone deacetylase regulation of ATM-mediated DNA damage signaling. Mol Cancer Ther 12: 2078-2087, 2013.

65. Le Corre L, FustierP, Chalabi N,Bignon YJ and Bernard-Gallon D: Effects of resveratrol on the expression of a panel of genes interacting with the BRCA1 oncosuppressor in human breast cell lines. Clin Chim Acta 344: 115-121, 2004.
66. Matsuda S, Nakagawa Y, Kitagishi Y, Nakanishi A and Murai T: Reactive oxygen species, superoxide dimutases, and PTEN-p53-AKT-MDM2 signaling loop network in mesenchymal Stem/Stromal cells regulation. Cells 7: 36, 2018.

67. Hair JM, Terzoudi GI, Hatzi VI, Lehockey KA, Srivastava D, Wang W, Pantelias GE and Georgakilas AG: BRCA1 role in the mitigation of radiotoxicity and chromosomal instability through repair of clustered DNA lesions. Chem Biol Interact 188: 350-358, 2010.

68. Liu W, Zhou Y, Reske SN and Shen C: PTEN mutation: Many birds with one stone in tumorigenesis. Anticancer Res 28: 3613-3619, 2008.

69. Kobayashi H, Ogawa K, Kawahara N, Iwai K, Niiro E, Morioka S and Yamada Y: Sequential molecular changes and dynamic oxidative stress in high-grade serous ovarian carcinogenesis. Free Radic Res 51: 755-764, 2017.

70. Bankoglu EE, Tschopp O, Schmitt J, Burkard P, Jahn D, Geier A and Stopper H: Role of PTEN in oxidative stress and DNA damage in the liver of whole-body Pten Haplodeficient mice. PLoS One 11: e0166956, 2016.

71. Armstrong CW, Maxwell PJ, Ong CW, Redmond KM, McCann C, Neisen J, Ward GA, Chessari G, Johnson C, Crawford NT, et al: PTEN deficiency promotes macrophage infiltration and hypersensitivity of prostate cancer to IAP antagonist/radiation combination therapy. Oncotarget 7: 7885-7898, 2016

72. Zhang R, Zhu L, Zhang L, Xu A, Li Z, Xu Y, He P, Wu M, Wei F and Wang C: PTEN enhances G2/M arrest in Etoposide-treated MCF-7 cells through activation of the ATM pathway. Oncol Rep 35: 2707-2714, 2016.

73. Yin H, Zhou Y, Wen C, Zhou C, Zhang W, Hu X, Wang L, You C and Shao J: Curcumin sensitizes glioblastoma to temozolomide by simultaneously generating ROS and disrupting AKT/mTOR signaling. Oncol Rep 32: 1610-1616, 2014.

This work is licensed under a Creative Commons Attribution-NonCommercial-NoDerivatives 4.0 International (CC BY-NC-ND 4.0) License. 